\title{
Final-state interactions in the superscaling analysis of neutral-current quasielastic neutrino scattering
}

\author{
M. C. Martínez, ${ }^{1}$ J. A. Caballero, ${ }^{2}$ T. W. Donnelly, ${ }^{3}$ and J. M. Udías ${ }^{1}$ \\ ${ }^{1}$ Grupo de Física Nuclear, Departamento de Física Atómica, Molecular y Nuclear, Universidad Complutense de Madrid, \\ E-28040 Madrid, Spain \\ ${ }^{2}$ Departamento de Física Atómica, Molecular y Nuclear, Universidad de Sevilla, Apdo. 1065, E-41080 Sevilla, Spain \\ ${ }^{3}$ Center for Theoretical Physics, Laboratory for Nuclear Science and Department of Physics, Massachusetts Institute of Technology, \\ Cambridge, Massachusetts 02139, USA
}

(Received 27 March 2008; published 6 June 2008)

\begin{abstract}
Effects of strong final-state interactions in the superscaling properties of neutral-current quasielastic neutrino cross sections are investigated by using the relativistic impulse approximation as guidance. First- and second-kind scaling are analyzed for neutrino beam energies ranging from 1 to $2 \mathrm{GeV}$ for the cases of ${ }^{12} \mathrm{C},{ }^{16} \mathrm{O}$, and ${ }^{40} \mathrm{Ca}$. Different detection angles of the outgoing nucleon are considered to sample various nucleon energy regimes. Scaling of the second kind is shown to be very robust. The validity of first-kind scaling is found to be linked to the kinematics of the process. Superscaling still prevails even in the presence of very strong final-state interactions, provided that some kinematical restrains are kept, and the conditions under which superscaling can be applied to predict neutral-current quasielastic neutrino scattering are determined.
\end{abstract}

DOI: 10.1103/PhysRevC.77.064604

PACS number(s): 25.30.Pt, 13.15.+g, 24.10.Jv

\section{INTRODUCTION}

Accurate predictions for neutrino-nucleus cross sections are needed in the analyses of ongoing and future experimental studies of neutrino reactions and neutrino oscillations [1] at intermediate energies. One option is to rely on direct modeling of the neutrino-nucleus interaction. To date this is the choice for essentially all neutrino event generators employed in accelerator and astroparticle neutrino experiments, where the relativistic Fermi gas (RFG) is usually incorporated as a standard tool. Proceeding in this way, one should keep in mind that any model for neutrino-induced reactions should first succeed in comparisons with the available high-quality inclusive electron cross section data, since typically the various semileptonic cross sections are closely related. A large variety of models, including the RFG, are not successful in reproducing electron scattering data when agreement to better than $20 \%-30 \%$ is desired. To avoid the nuclear uncertainties inherent in any neutrino-nucleus reaction description, the authors in Ref. [2] have proposed the idea of profiting from the extensive knowledge on nuclear dynamics acquired from electron scattering experiments to predict inclusive chargedcurrent neutrino-nucleus cross sections. The connection between the two electroweak processes is done by means of the superscaling analysis. The successful application of the phenomenological superscaling approach (SuSA) [2-12] has motivated us to revisit the concept of scaling and study its validity when applied to neutral-current neutrino cross sections.

It is very well established that the large amount of inclusive electron scattering data manifests scaling behavior at high momentum transfer for excitation energies falling below the quasielastic $(\mathrm{QE})$ peak [13-17]. These $\left(e, e^{\prime}\right)$ data (particularly those coming from the analysis of the longitudinal response), when appropriately organized, show reasonably good scaling of the first kind (no dependence on the momentum transfer $q$ ) and excellent scaling of the second kind (no dependence on the particular nuclear species). They are said to superscale. As an outcome of this behavior, a phenomenological scaling function has been directly derived from the longitudinal data $[14,16]$. This scaling function contains the relevant information about the initial- and final-state nuclear dynamics explored by the probe, in this case, an electron. Recently, the scaling analysis has also been extended to inelastic responses in the region of the delta peak and even beyond [2,18], and scaling ideas have also been applied to processes involving hadronic probes [19]. It is important to notice that superscaling is a general phenomenon exhibited by nature (although not perfectly). Essentially it shows up upon dividing the electron-nucleus cross section data at sufficiently high $q$ by the corresponding electron-nucleon cross section, and plotting the results against a properly chosen scaling variable, which can be derived by using simple kinematical considerations. Superscaling of data is thus independent of any modeling of the reaction, although it is a characteristic of the inclusive electron scattering that any "reliable" model should be able to reproduce. For instance, it is well known that, although the relativistic Fermi gas exhibits perfect superscaling [20] (and has even inspired a particular scaling variable), the scaling function it predicts differs from the experimental result. In fact, only a few models to date can accurately reproduce the experimental $\left(e, e^{\prime}\right)$ scaling function. One of these is based on the relativistic impulse approximation when strong relativistic mean-field potentials are used to describe the bound and ejected nucleon wave functions. This point will be discussed at length later.

The kinematics involved in $\left(e, e^{\prime}\right)$ and $(\nu, \mu)$ reactions are rather similar; that is, in both cases the scattered lepton is detected, and both the energy and momentum transferred by the probe to the nucleus are thus known. Accordingly, it is plausible to expect that the two probes explore the nucleus in a similar way, and consequently both electron 
and charged-current neutrino scattering share the same universal scaling function. This is the main approximation adopted by SuSA, where the scaling function is determined by using longitudinal electron scattering data and then carried forward to make predictions for neutrino-induced processes [2,9]. These phenomenological predictions, which incorporate nuclear information provided directly by the analysis of $\left(e, e^{\prime}\right)$ experimental data, are believed to be more robust and "reliable" than those coming from direct modeling.

A couple of questions arise naturally. Can these superscaling ideas also be applied to neutral-current neutrino-nucleus cross sections? Can we get reliable predictions for neutralcurrent processes based on the phenomenological electron superscaling function? These neutrino processes play an important role in the determination of the strange quark contribution to the nucleon spin. Furthermore, neutral-current reactions are also relevant for oscillation experiments-for instance, it is expected that they contribute as the third most important event type for the MiniBooNE experiment at Fermilab [1]. As in the case of charged-current processes, neutral-current neutrino-nucleus cross section predictions based on scaling ideas, when possible, are clearly demanded. However, the extension of the scaling analysis and the application of SuSA ideas to neutral-current reactions is not as straightforward as in the case of charged-current processes. Focusing on the quasielastic region, in the case of neutral-current (NC) processes, one finds that the scattered neutrino is not detected and identification of the NC event may be made by observing a nucleon ejected from the nucleus without finding evidence of final charged leptons. Even if one measures the nucleon energy and momentum, the transferred energy and momentum at the leptonic vertex will remain unknown. Hence, the NC kinematics are clearly different from those corresponding to electron and charged-current neutrino scattering processes. This makes the applicability of scaling arguments to NC reactions less obvious [11]. Given the basic differences in the two types of kinematics, it is first necessary to establish the validity of scaling ideas for NC neutrino scattering. Second, the phase-space regions explored in the two processes (NC versus electron and charged-current neutrino scattering) are different, and they could display distinct sensitivities to the many-body physics underlying the scaling function. Thus, the idea of using the experimental electron scaling function to predict NC neutrino cross sections, as was done for charged-current processes, requires a more in-depth analysis. In other words, prior to any extension of SuSA analyses to predict $\mathrm{NC}$ neutrino scattering in the quasielastic region, one needs to be sure whether superscaling also holds for NC neutrino-nucleus scattering, and moreover, whether the phenomenological $\left(e, e^{\prime}\right)$ scaling function can safely be used to predict $\mathrm{NC}$ cross sections, in spite of the intrinsic differences between the two processes.

To proceed, the ideal strategy would be to count on sufficient NC neutrino-nucleus experimental data to perform a scaling analysis similar to the one used for the electron case, and compare the NC scaling function (if any) with the electron one. Unfortunately, $\mathrm{NC}$ data are too scarce to allow for a meaningful scaling analysis, and one has to rely on neutrino- nucleus models that are expected to mimic the behavior that the data should show.

Keeping this in mind, the natural starting point for examining scaling properties of NC neutrino scattering is the relativistic Fermi gas. This model is known to exhibit perfect superscaling when applied to inclusive quasielastic electron and charged-current neutrino scattering. Such a study was recently undertaken for NC neutrino reactions in Ref. [11], where it was shown how to extend the scaling ideas to NC processes (within the RFG model), illustrating the results for scattering of $1-\mathrm{GeV}$ neutrinos from ${ }^{12} \mathrm{C}$. Additionally, the universality property of the scaling function was assumed in Ref. [11], and hence NC neutrino cross sections were predicted by making use of the averaged phenomenological scaling function extracted from the analysis of $\left(e, e^{\prime}\right)$ data. The same idea of universality of the scaling function was exploited in Ref. [12], where the coherent density fluctuation model scaling function was used to predict NC neutrino cross sections, and results were compared with those based on the phenomenological $\left(e, e^{\prime}\right)$ scaling function. A first obstacle to assessing the likelihood of being able to predict NC cross sections in this way is that the RFG, although providing a good framework to start with, is surely too simple to describe satisfactorily the behavior showed by $\mathrm{NC}$ data if uncertainties of less than $20 \%-30 \%$ are desired. Although the RFG $\left(e, e^{\prime}\right)$ response by definition exhibits perfect superscaling [20], it lacks important dynamical effects, hence providing responses that are not fully in accord with the magnitude and shape of the experimental scaling function. Indeed, it has been shown that strong final-state interactions (FSI) are needed to describe successfully the magnitude and shape of the superscaled data, introducing small deviations from perfect superscaling behavior as well. It is expected that FSI also modify the NC neutrino-nucleus cross sections in a significant way, and thus it is necessary to infer how FSI may affect the scaling properties.

In this work, which is complementary to that in Ref. [22], we address this issue: We perform a systematic analysis of the effects of strong FSI in the superscaling properties of quasielastic NC neutrino-nucleus scattering within the context of the relativistic impulse approximation (RIA), based on strong relativistic mean-field (RMF) potentials for both the bound and the ejected nucleons. Several arguments motivate our choice of this particular model to illustrate the possible effects of FSI. The RIA model has been extensively and successfully applied in investigations of exclusive electron scattering reactions [23]. Furthermore, the RIA approach has been shown to superscale when applied to quasielastic inclusive electron and charged-current neutrino scattering, giving rise to a "unique" scaling function, with relatively mild scaling violations. This result reinforces the idea of the existence of a universal scaling function that is valid for both electron and charged-current neutrino probes [3,5]. Finally, the most important reason for the choice of the RIA, as well as its corresponding semirelativistic version [6], is that it is capable of reproducing the shape and magnitude of the experimental scaling curve extracted from QE $\left(e, e^{\prime}\right)$ data, something that has proven to be elusive for other theoretical models. In particular, the asymmetric shape exhibited by the experimental scaling function with a significant tail extending to high values of the 
transfer energy is accurately reproduced by the RIA, provided that FSI are described with the RMF approach [3,5]. For all these reasons, and, being aware of the scarcity of NC neutrino data, we are led to accept the validity of the RIA with FSI treated through the use of the RMF as a realistic model for the description of inclusive scattering and believe that it can serve as a reliable tool to illustrate possible FSI effects on the scaling properties of quasielastic NC neutrino cross sections.

The paper is organized as follows. In Sec. II we present a brief summary of the formalism required to treat $\mathrm{NC}$ neutrino-nucleus scattering in the RIA. A general discussion of the superscaling phenomenon is also provided, showing the basic expressions to be used in the analysis. In Sec. III we discuss the results. First, we focus on differential cross sections and response functions for neutrino- and antineutrino-induced proton and neutron knockout. Several kinematical situations of interest are examined. Second, we evaluate the scaling function and perform a separate study of the scaling properties (scaling of the first and second kinds), paying special attention to FSI effects. Results are illustrated for various choices of kinematics and for three nuclei, ${ }^{12} \mathrm{C},{ }^{16} \mathrm{O}$, and ${ }^{40} \mathrm{Ca}$. A comparison between the RIA-RMF NC and the phenomenological electron scaling functions is also performed. Finally, in Sec. IV we summarize our main conclusions.

\section{FORMALISM FOR NEUTRAL-CURRENT NEUTRINO-NUCLEUS SCATTERING}

Following the general procedure for superscaling analyses, we start by evaluating inclusive $\mathrm{QE}$ neutral-current neutrino cross sections within the RIA model. We assume the inclusive cross section to be given as the integrated semi-inclusive one-nucleon (proton or neutron) knockout cross sections. This approximation, which is implicit in scaling analyses, has been shown to work successfully in the kinematic region dominated by quasielastic scattering. In other words, we construct the inclusive $A(v, N) v^{\prime} A-1$ cross section, within the RIA model, by integrating the $A\left(v, v^{\prime} N\right) A-1$ cross section over the unobserved scattered neutrino variables.

\section{A. Relativistic impulse approximation}

The RIA model has been used to describe NC neutrinonucleus reactions in previous work [24,25]. Here we simply summarize those aspects that are of most relevance for later discussion of the scaling properties.

The first basic assumption of the RIA is that the process occurs through the exchange of a single vector boson; this is known as the first Born approximation. In this approach, the leading-order exclusive quasielastic cross section is generated by the Feynman amplitude associated with the diagram shown in Fig. 1. Here, a neutrino scatters off an $A$-body nucleus via the exchange of a $Z^{0}$. In the scattering process, a nucleon is knocked out, leaving behind an $(A-1)$-body daughter nucleus, generally in an excited state. The kinematical variables can be inferred from the figure.

The RIA also assumes the impulse approximation; that is, the incident neutrino interacts with only one nucleon, which



FIG. 1. Feynman diagram for quasielastic NC neutrino-nucleus scattering in the first Born approximation

is subsequently emitted. The nuclear current is written as a sum of single-nucleon currents. Then, the transition matrix elements from which the cross section is computed can be cast in the following form:

$$
\left\langle J^{\mu}\right\rangle=\int d \mathbf{r} \bar{\phi}_{F}(\mathbf{r}) \hat{J}^{\mu}(\mathbf{r}) e^{i \mathbf{q} \cdot \mathbf{r}} \phi_{B}(\mathbf{r}),
$$

where $\phi_{B}$ and $\phi_{F}$ are relativistic bound-state and scattering wave functions, respectively, and $\hat{J}^{\mu}$ is the relativistic onebody current operator modeling the coupling between the virtual $Z^{0}$ and a bound nucleon. (See Refs. [24,25] for details concerning the operator and nucleon form factors; in all results presented in the next section we have not allowed for strangeness content in the nucleon.) We describe the bound nucleon states as self-consistent Dirac-Hartree solutions, derived within a RMF approach by using a Lagrangian containing $\sigma, \omega$, and $\rho$ mesons [26].

Ignoring all distortions from FSI leads to the description of the scattering wave function for the outgoing nucleon as a relativistic plane wave. This is known as the relativistic plane-wave impulse approximation (RPWIA) [27,28], which obviously entails a simplified description of the reaction mechanism. In contrast, in the present work, when accounting for FSI between the ejected nucleon and the residual nucleus, the outgoing nucleon wave function is computed by using the same relativistic mean field (RMF) employed to describe the initial bound states. We denote this approach as RMF [3,5,24,25,29,30].

Using these ingredients, we evaluate the six-differential cross section $d^{6} \sigma / d \varepsilon^{\prime} d \Omega_{k^{\prime}} d E_{N} d \Omega_{N}$. The inclusive cross section is obtained by integrating over the three momenta of the undetected particles. In NC neutrino scattering the outgoing nucleon is assumed to be the only particle detected in the final state; hence one integrates over the scattered neutrino variables $\varepsilon^{\prime}$ and $\Omega_{k^{\prime}}$. A sum over all single-particle states from which the nucleon may originate is also performed.

As can be inferred from the way we are proceeding, processes containing other particles in addition to the nucleon in the final state, including multinucleon knockout and pion production, are not explicitly taken into account. Within the context of the RMF (mean-field-based model) only one-body processes are explicitly considered and the inclusive strength is exclusively built out of impulse approximation single-nucleon 
knockouts. Note, however, that the one-body contribution from multinucleon knockout is fully incorporated into the RMF model, via the self-energy of the propagating nucleon. In other words, the RMF approach includes all kind of rescattering (elastic and inelastic) with the remaining nucleons. Thus, in this mean-field picture the redistribution of the strength and multinucleon knockout are completely attributed to FSI and not to explicit correlations, which are in fact incorporated within the RMF in an effective way. Notice that, owing to the presence of strong scalar and vector potentials combining repulsive and attractive interactions that provide the correct saturation properties for nuclear matter, the RMF has more flexibility to incorporate correlations in an effective way than does the nonrelativistic mean-field approach [31].

The adequacy of the potential to be employed for inclusive scattering has been investigated in several works [32-35]. Besides the RMF, approaches where one uses only the real part of the optical potential [29], or where the full optical potential is employed but a formal summation based on Green function formalism over all possible channels that contribute to the inclusive scattering is done [32,34,35], have been used to describe lepton-nucleus scattering under inclusive conditions. The comparison of results from these approaches show that they differ by a few percent and in general they compare equally well to existing electron scattering data. Thus, in this work we present only results obtained within the relativistic mean field.

\section{B. Scaling and superscaling analysis}

In the case of electron and charged-current neutrino scattering, the general procedure to get the scaling function is to divide the inclusive differential cross section by the single-nucleon $e N$ or $v N$ cross section, weighted by the number of protons and neutrons involved in the process. If the function obtained in this way, when plotted against a properly chosen variable (the scaling variable), is seen to depend weakly on the momentum transfer, one observes scaling of first kind. Additionally, both the scaling function and the scaling variable can be made dimensionless via a characteristic momentum scale for the chosen nucleus. If the scaling function so obtained does not depend on the particular nuclear species involved, one talks about scaling of the second kind. When both types of scaling occur, the data or the calculations are said to superscale. One should notice the important role played by the kinematics in the general derivation of scaling for both electron and charged-current neutrino scattering. Two factors seem to be essential for scaling to occur: (i) The transferred four-momentum $Q^{\mu}$ is fixed in the process, and (ii) the missing-energy missing-momentum region accessible to the reaction is relatively confined. This is the situation for the $t$-channel processes (electron and charged-current neutrino scattering).

Being aware of the essential differences mentioned in the introduction between the kinematics involved in inclusive electron or charged-current neutrino reactions and those for NC neutrino processes, we start the present study in the RIA by reviewing briefly the general discussion on the QE neutral-current kinematics and scaling properties presented in Refs. [11,21]. Summarizing, for NC scattering we assume the energy $\varepsilon$ of the incident neutrino to be specified (in real situations there are usually no monochromatic beams and an integral over the allowed energies folded with the neutrino flux must be performed) and also assume the outgoing nucleon energy $E_{N}$ to be known. Finally, the angle $\theta_{k p_{N}}$ between the incident neutrino and the ejected nucleon momentum is also given. Notice that, in contrast to electron and charged-current neutrino reactions, the scattered lepton four-momentum $Q^{\mu}=$ $K^{\mu}-K^{\prime \mu}$ is not fixed, making the analysis of the scaling behavior more difficult. As already presented in Ref. [11], one can introduce a corresponding constant Lorentz invariant $Q^{\prime 2}=\left(K^{\mu}-P_{N}^{\mu}\right)^{2}=\omega^{\prime 2}-q^{\prime 2}$, where $\omega^{\prime}=\varepsilon-E_{N}$ and $q^{\prime}=\left|\mathbf{q}^{\prime}\right|=\left|\mathbf{k}-\mathbf{p}_{N}\right|=\sqrt{\varepsilon^{2}+p_{N}^{2}-2 \varepsilon p_{N} \cos \theta_{k p_{N}}}$.

In Ref. [11] the influence of a nonconstant $Q^{\mu}$ in the derivation of scaling in the NC case was thoroughly investigated, with the conclusion within the general framework of the relativistic Fermi gas model that scaling ideas still work properly for NC neutrino-nucleus processes. In this work we shall not repeat that study, but rather we take it as our starting point. Our main aim here is to go a step further by analyzing whether or not the presence of strong FSI affects the scaling properties of QE neutral-current cross sections. Inspired by the RFG result, in this work we construct the following function from the inclusive NC neutrino cross section evaluated in the RIA:

$$
f\left(q^{\prime}, \psi^{u}\right) \equiv k_{A} F\left(q^{\prime}, \psi^{u}\right)=k_{A} \frac{\left[\frac{d \sigma}{d \Omega_{N} d p_{N}}\right]}{\bar{\sigma}_{\mathrm{sn}}^{\mathrm{NC}}},
$$

where $\bar{\sigma}_{\mathrm{sn}}^{\mathrm{NC}}$ is the effective NC single-nucleon cross section (given explicitly in Eq. (20) and the appendix in Ref. [11]), and $\psi^{u}\left(q^{\prime}, \omega^{\prime}\right)$ is the dimensionless scaling variable extracted from the RFG analysis to be used for QE neutral-current kinematics (see Eqs. (24)-(26) in Ref. [11]). The momentum $k_{A}$ introduced in Eq. (2) states a characteristic momentum scale for a given nuclear species, allowing one to define the dimensionless scaling function $f\left(q^{\prime}, \psi^{u}\right)$. This scaling function can be plotted versus $\psi^{u}$ for different neutrino beam energies (which at fixed $\theta_{k p_{N}}$ and given $E_{N}$ means different $q^{\prime}$ values) and different nuclear species, thereby yielding a way of analyzing first- and second-kind scaling.

It is important to note that the azimuthal angle $\phi_{N}$ between the outgoing nucleon and the scattering plane (which contains both the incident and the scattered neutrinos) will not cover the full range from 0 to $2 \pi$ (see the discussion in Ref. [11]), and hence there will be nonzero contributions in the inclusive $\mathrm{NC}$ cross sections with detection of the outgoing nucleon ( $T L, T T$, and $T L^{\prime}$ terms), which would vanish in the usual inclusive charged-current neutrino and electron scattering reactions.

\section{RESULTS}

\section{A. Differential cross sections and responses}

We begin by evaluating final-state interaction effects on inclusive QE neutral-current neutrino scattering. We first focus on the case of ${ }^{12} \mathrm{C}$, whose choice is motivated not only by the relevance for present neutrino oscillation experiments but 


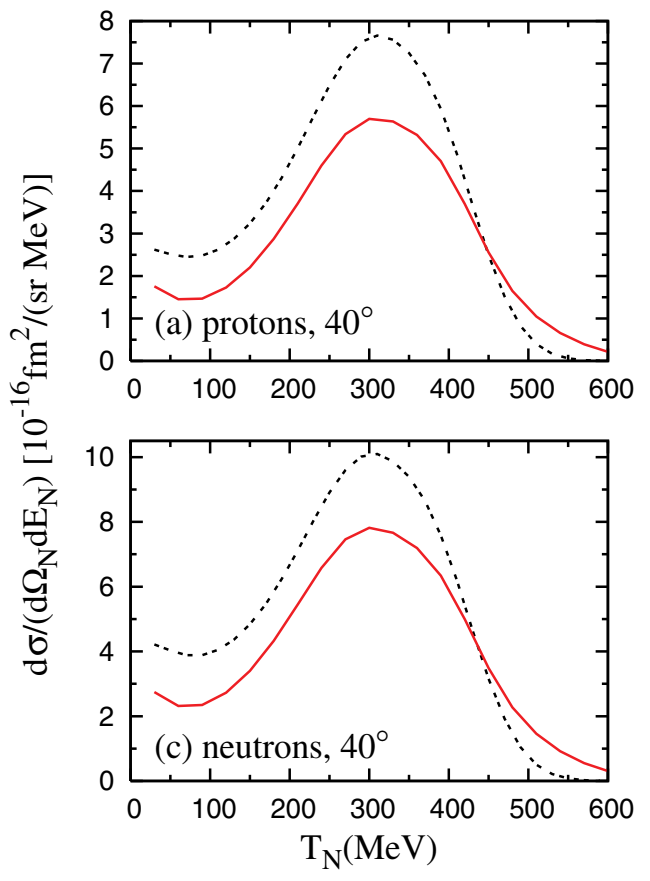

also because it facilitates the comparison with the relativistic Fermi gas results presented in Ref. [11]. In Fig. 2 we present the differential cross section $d \sigma / d E_{N} d \Omega_{N}$ at incident neutrino energy $\varepsilon=1 \mathrm{GeV}$ for ${ }^{12} \mathrm{C}(v, p)$ and ${ }^{12} \mathrm{C}(v, n)$ as a function of the outgoing nucleon kinetic energy $T_{N}$. The two curves in each panel correspond to different descriptions of the outgoing nucleon wave function: RPWIA (dashed) and RMF potential (solid). Results are shown for two representative values of the nucleon scattering angle, $40^{\circ}$ [Figs. 2(a) and 2(c)] and $60^{\circ}$ [Figs. 2(b) and 2(d)].

Final-state interactions lead to a decrease of the cross sections. Furthermore, RMF results exhibit an increase of strength at large $T_{N}$. The existence of this longer tail within the RMF approach is due to the strong relativistic potentials used in describing the final-state interaction. This is consistent with what was already observed and discussed within the context of $\left(e, e^{\prime}\right)$ and charged-current neutrino-nucleus cross sections, where a pronounced tail extending to small final-state lepton kinetic energies also emerges from the use of the RMF potential $[3,5]$. The magnitude of the scalar and vector potentials in the RMF, which are energy independent, can shift significant strength to higher nucleon kinetic energies [3,5]. This effect of the strong RMF potential is the one that provides the theoretical $\left(e, e^{\prime}\right)$ and $(\nu, \mu)$ RIA superscaling functions with the correct asymmetry, namely, the one observed in the experimental data at high energy transfers (positive values of the scaling variable).

As shown in Fig. 3, FSI have a similar influence on antineutrino proton and neutron knockout cross sections. Notice that $\bar{v}$ cross sections are somewhat smaller, particularly for the lower values of $\theta_{k p_{N}}$ (forward scattering). Second, the long tail displayed for low $T_{N}$ and observed at $\theta_{k p_{N}}=40^{\circ}$ is significantly more pronounced in the antineutrino case. Note that the cross sections even change their behavior in this region; they increase significantly as $T_{N}$ approaches zero.
FIG. 2. (Color online) QE differential cross sections for $\mathrm{NC}$ neutrino scattering at $1 \mathrm{GeV}$ from ${ }^{12} \mathrm{C}$ for proton [(a) and (b)] and neutron [(c) and (d)] knockout. Panels (a) and (c) correspond to $\theta_{k p_{N}}=40^{\circ}$ and panels (b) and (d) to $60^{\circ}$. Results are given for the RPWIA and the RMF-FSI descriptions.
In accordance with what was observed within the context of the RFG in Ref. [11], a common feature of the two models, RPWIA and RMF, is that the differential cross sections present a similar shape for both proton and neutron emission, although with somewhat different magnitude. Neutron ejection involves larger cross sections, as one expects from the isovector dependence of the coupling to the $Z^{0}$ [11].

The separate response functions contributing to the RPWIA cross sections in Figs. 2 and 3 are displayed in Fig. 4. These RPWIA responses are very similar to those presented for the relativistic Fermi gas model in Ref. [11] at $\theta_{k p_{N}}=60^{\circ}$ (see Fig. 6 in the earlier reference). This result is indeed consistent with the minor role of nuclear modeling expected at energies of the order of $1 \mathrm{GeV}$ and higher [29]. Thus, most of the features concerning the responses at $60^{\circ}$ signaled in Ref. [11] apply also to our present results within the relativistic impulse approximation: The purely transverse $T$ and $T^{\prime}$ responses yield the dominant contributions. In the case of neutron emission, the purely longitudinal $L$ response also produces a significant contribution, particularly for small values of the nucleon kinetic energy. The $T L$ interference response also contributes to the shape and magnitude of the cross sections. This applies not only to the $60^{\circ}$ results but also to the $40^{\circ}$ ones. Finally, the $T T$ and $T L^{\prime}$ response functions have an almost negligible effect on the cross section for all cases.

Although not shown for brevity, the RMF responses have similar trends to those for the RPWIA case, but with reduced magnitudes. In particular, responses evaluated with the RMF potential vanish at higher $T_{N}$, in accordance with the behavior of the cross sections shown in Fig. 2.

Let us now turn to the main goal of this section, namely describing the behavior of the QE neutral-current neutrino cross sections with respect to changes in the kinematics. This is crucial for understanding the outcomes of the scaling analysis discussed in the next section. The results in Fig. 5 


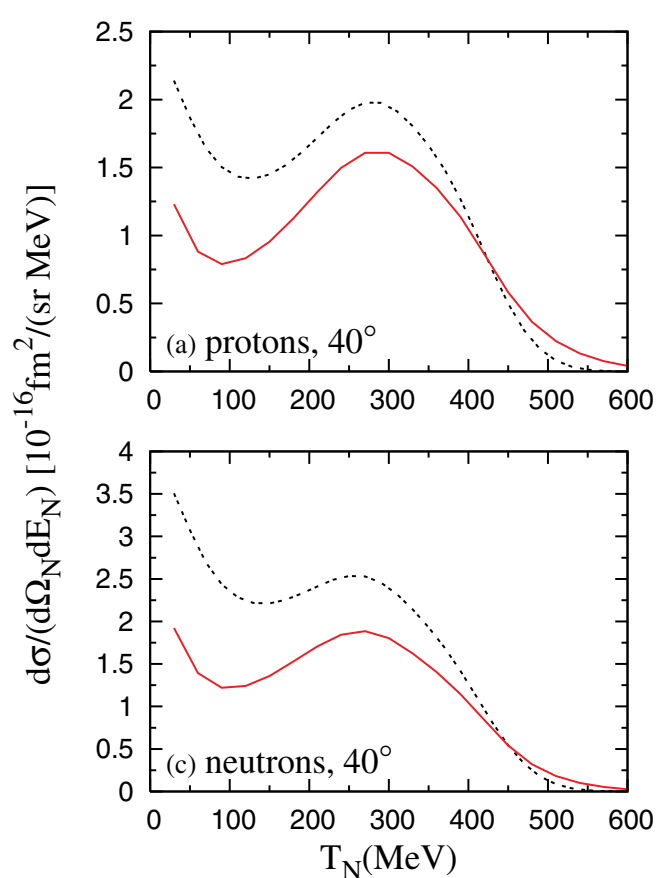

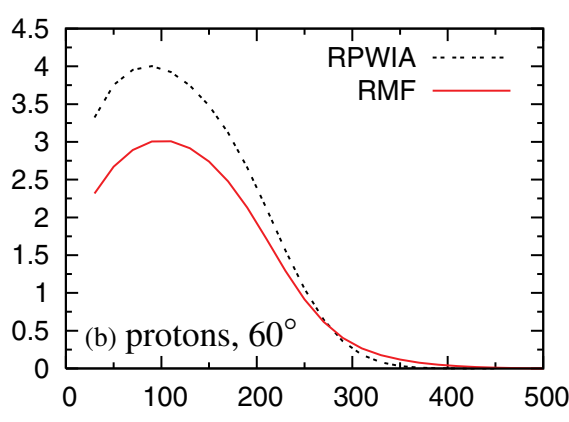



FIG. 3. (Color online) The same plots as in Fig. 2, but now for antineutrinos. correspond to ${ }^{12} \mathrm{C}(v, p)$ differential cross sections within the RMF for three neutrino beam energies, $1,1.5$, and $2 \mathrm{GeV}$ [Figs. 5(a), 5(b), and 5(c), respectively]. For each energy, we study the variation of the cross sections with the nucleon scattering angle. Two observations can be made based on the figure:

(i) For a given neutrino energy, $\theta_{k p_{N}}$ determines the value of the nucleon kinetic energy for which the cross section presents its maximum. In general, small angles correspond to cross sections peaked at higher values of kinetic energy. Hence, changing the value of $\theta_{k p_{N}}$ allows us to explore different regions of nucleon kinetic energy.

(ii) For a given $\theta_{k p_{N}}$, changing the neutrino beam energy means changing the position and magnitude of the maximum of the cross section. This change is significantly stronger at more forward angles.

The evolution of the QE neutral-current ${ }^{12} \mathrm{C}(v, p)$ cross section can be easily understood from the consideration of the corresponding cross section from free nucleons at rest. In such a case, energy and momentum conservation imposes a relationship among the values of $\theta_{k p_{N}}$, the angle of the nucleon

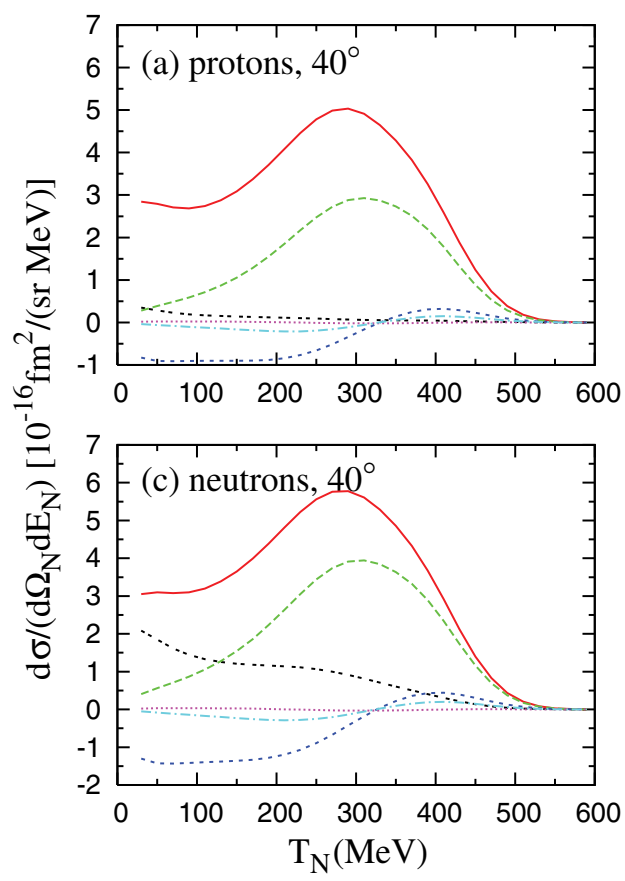



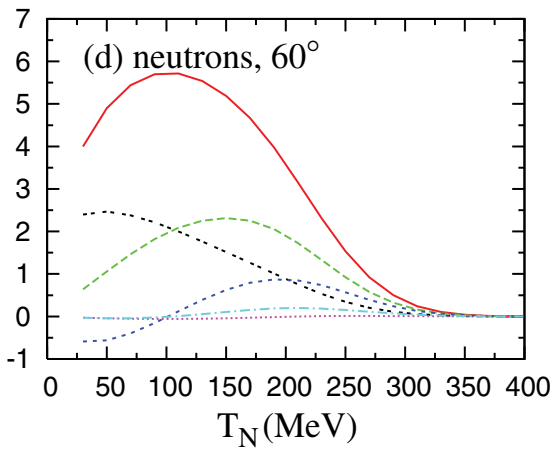

FIG. 4. (Color online) Response functions contributing to the RPWIA cross sections in Fig. 2 for proton [(a) and (b)] and neutron [(c) and (d)] knockout. Panels (a) and (c) correspond to $\theta_{k p_{N}}=40^{\circ}$ and panels (b) and (d) to $\theta_{k p_{N}}=60^{\circ}$. 


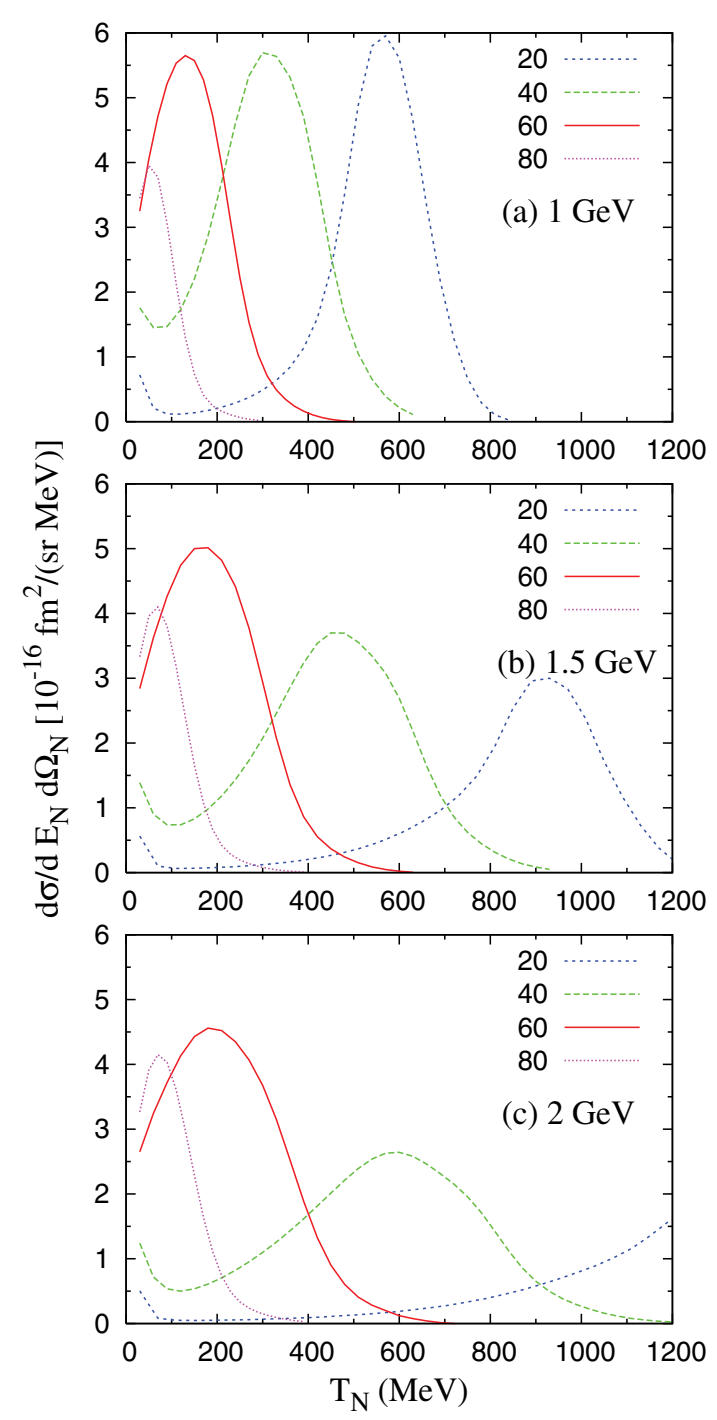

FIG. 5. (Color online) RMF differential cross sections for the $\mathrm{NC}{ }^{12} \mathrm{C}(v, p)$ reaction at various beam energies and detection angles of the outgoing proton, $\theta_{k p_{N}}$. Panels (a), (b), and (c) correspond to $\varepsilon=1,1.5$, and $2 \mathrm{GeV}$, respectively. The different curves in each panel are obtained by changing the value of $\theta_{k p_{N}}$ from $20^{\circ}$ to $80^{\circ}$, as specified in the label.

with respect to the incident neutrino beam, and $T_{N}$, the energy of the final nucleon. This relation is given in the form

$$
T_{N}=\frac{2 M_{N} \varepsilon^{2} \cos ^{2} \theta_{k p_{N}}}{\left(\varepsilon+M_{N}\right)^{2}-\varepsilon^{2} \cos ^{2} \theta_{k p_{N}}} .
$$

Results from Eq. (3) for several values of the incoming neutrino energy, $\varepsilon$, are shown in Fig. 6. For bound nucleons, apart from the effect of Fermi motion inside the nuclei that broadens the peak and shifts it a bit with regard to the free-nucleon case, we expect the maxima of the cross sections to be located approximately where Eq. (3) predicts (see Fig. 6). This explains in a simple way our observations about the QE neutral-current ${ }^{12} \mathrm{C}(v, p)$ cross section. These results are thus directly linked to the kinematics of the process, and not to the use of the RIA-RMF model. In particular, the fact that the

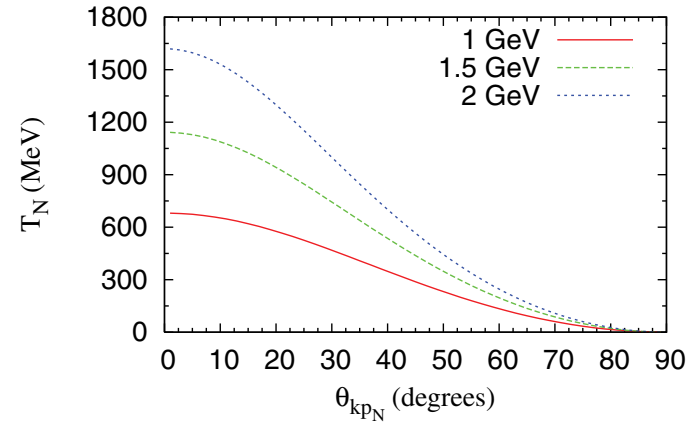

FIG. 6. (Color online) Relationship between the detection angle of the outgoing proton and the value of the proton kinetic energy for the NC $(v, p)$ reaction on free protons at rest. The different curves show the results for beam energies of $1,1.5$, and $2 \mathrm{GeV}$.

range of $T_{N}$ spanned at fixed $\theta_{k p_{N}}$ for varying beam energy is reduced for large angles as seen in Figs. 5 and 6 will have important implications in the first-kind scaling properties of the cross sections.

\section{B. Scaling analysis}

In this section we present a general study of the scaling properties of the QE neutral-current neutrino cross sections with strong FSI. Our aim is twofold. First, we investigate whether FSI effects may (or not) prevent the appearance of superscaling. Second, if superscaling holds even in this case, we study the conditions under which the SuSA approach [based on the experimental superscaling $\left(e, e^{\prime}\right)$ response] can be used to predict NC neutrino-nucleus cross sections. As stated in the introduction, we use the RIA-RMF model to illustrate the results, although our findings are not intrinsically linked to the use of this model, as will be clarified later.

To begin let us focus on the first-kind scaling analysis of the $(v, p)$ cross sections. In the top panels of Fig. 7 we present ${ }^{12} \mathrm{C}(\nu, p)$ quasielastic differential cross sections for different nucleon detection angles and beam energies. Each graph corresponds to a certain $\theta_{k p_{N}}$, from $20^{\circ}$ to $80^{\circ}$, and for each $\theta_{k p_{N}}$ value results for three neutrino beam energies, 1 , 1.5 , and $2 \mathrm{GeV}$, are displayed. The characteristic momentum for ${ }^{12} \mathrm{C}$ is taken to be $k_{A}=228 \mathrm{MeV} / c$, as suggested by the superscaling analysis of electron scattering in Ref. [16]. As already observed in Fig. 5 and explained from the simple consideration of free nucleons in the previous section, the variation of the cross sections is softer for angles equal to or larger than $60^{\circ}$. For $40^{\circ}$ and smaller there is a noticeable shift of the cross sections to higher nucleon kinetic energies, as well as a considerable variation in magnitude when the beam energy increases. If first-kind scaling holds, all of these cross sections should collapse to a unique curve when dividing by the NC single-nucleon cross section [see Eq. (2)] and plotting the results against the dimensionless scaling variable $\psi^{u}$. These results are shown in the bottom panels of Fig. 7. Focusing on larger angles, namely $60^{\circ}$ and $80^{\circ}$, one observes that scaling of the first kind is fulfilled to a high degree even in the presence of the very strong FSI included in the RIA-RMF model. In other words, the variations in the cross sections 

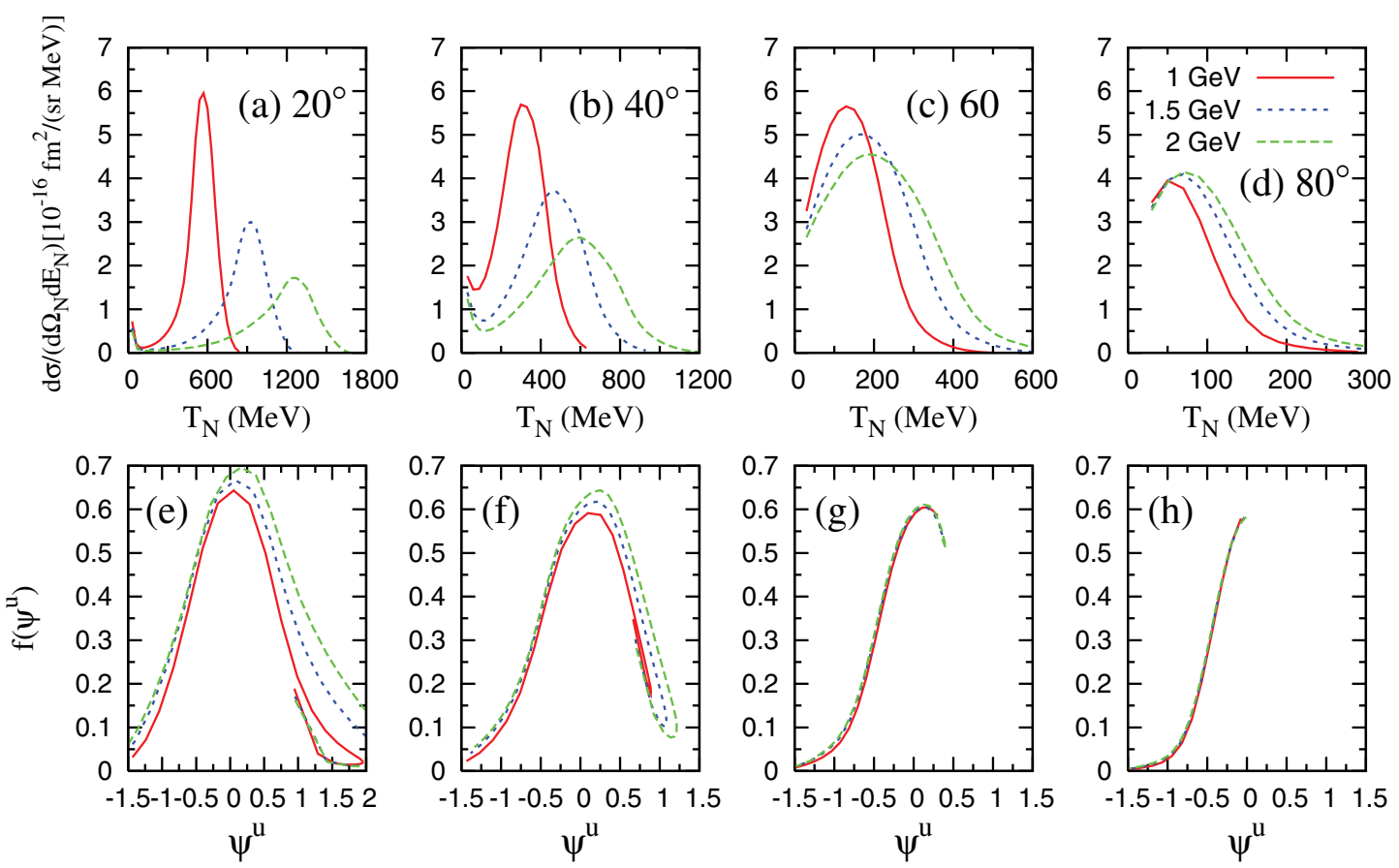

FIG. 7. (Color online) Differential cross section for ${ }^{12} \mathrm{C}(v, p)$ scattering in the RIA-RMF for different beam energies [(a), (b), (c), and (d)] and their corresponding scaling functions [(e), (f), (g), and (h)]. From left to right, the graphs correspond to different values of $\theta_{k p_{N}}$, namely $20^{\circ}, 40^{\circ}, 60^{\circ}$, and $80^{\circ}$.

observed for different neutrino energies are well accounted for by the single-nucleon part of the cross sections, which has been factored out in obtaining the scaling function. In contrast, for forward angles, $20^{\circ}$ and $40^{\circ}$, first-kind scaling is clearly not so well respected. Nevertheless, there are some features of first-kind scaling behavior that still persist for these forward angles: The peak of the scaling function appears approximately at the same $\psi^{u}$ value for all beam energies, and in the region of negative $\psi^{u}$ values first-kind scaling is reasonably well respected. However, the scaling functions obtained at $20^{\circ}$ and $40^{\circ}$ show an increase in the height of the peaks of the curves, as well as a shift to $\psi^{u}>0$ for increasing beam energy. This is similar to what is observed in the RIA-RMF for the inclusive $\left(e, e^{\prime}\right)$ case. Actually, the experimental $\left(e, e^{\prime}\right)$ data do leave room for some breaking of first-kind scaling in the region of a positive scaling variable.

A comment is in order concerning the behavior of the scaling function $f\left(\psi^{u}\right)$ for positive $\psi^{u}$ values. As noted, in this $\psi^{u}$ region $f\left(\psi^{u}\right)$ may be two-valued. This purely kinematical effect comes from the general expression for the $\psi^{u}$ variable and the kinematics involved in NC neutrino-nucleus scattering processes. This effect is present for all models whenever the kinematics lead to positive $\psi^{u}$ values close to or higher than 0.5 (most forward scattering angles). To clarify this point we present in Fig. 8 the behavior of the scaling variable $\psi^{u}$ at a beam energy of $1 \mathrm{GeV}$ as a function of the outgoing nucleon kinetic energy for four different values of the scattering angle, $\theta_{k p_{N}}=20^{\circ}, 40^{\circ}, 60^{\circ}$, and $80^{\circ}$. As shown, $\psi^{u}$ takes on a unique value for each $T_{N}$ for $\theta_{k p_{N}}=60^{\circ}$ and $80^{\circ}$. For lower scattering angles, there exists a region (wider for smaller $\theta_{k p_{N}}$ ) where two different values of the nucleon kinetic energy lead to the same $\psi^{u}$. Figure 8 also illustrates why the superscaling functions in Fig. 7 extend up to a different positive $\psi^{u}$ value depending on the nucleon scattering angle. In fact, this $\psi^{u}$ value, which corresponds to the minimum kinetic energy considered, is lower for increasing angles.

Up to now we have found that at forward scattering angles the results display a first-kind scaling violation to some extent, whereas at larger angles they exhibit almost perfect first-kind scaling. The origin of this scaling behavior of the QE neutral-current $(v, p)$ cross sections is easily understood when one realizes that first-kind scaling is very well fulfilled in the absence of FSI, as was also the case for electrons and charged-current neutrino reactions $[3,5,11,20]$. In Fig. 9 we present results analogous to those in Fig. 7, but now for the RPWIA. In contrast to what happens when FSI are accounted for, first-kind scaling is now well fulfilled independently of

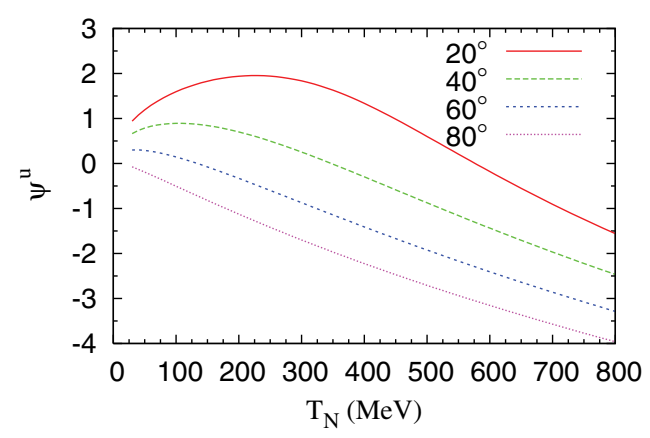

FIG. 8. (Color online) Behavior of the scaling variable $\psi^{u}$ as a function of the kinetic energy of the outgoing nucleon at $\varepsilon=1 \mathrm{GeV}$. 

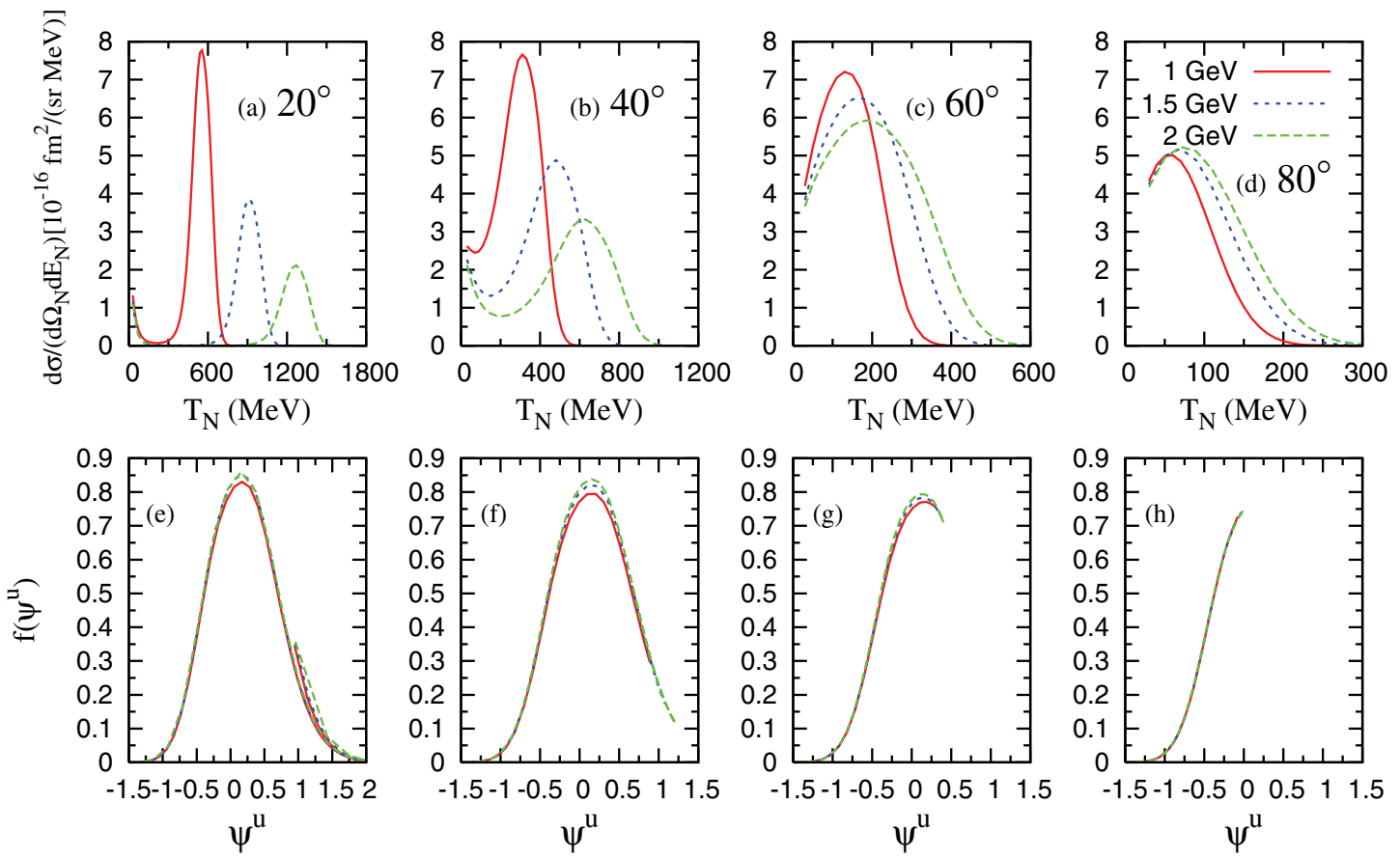

FIG. 9. (Color online) The same plots as in Fig. 7, but now for the RPWIA.

the value of $\theta_{k p_{N}}$. Therefore, the breakdown of first-kind scaling for forward angles in Fig. 7 must be ascribed to FSI. This may appear to be a little unusual when one examines the cross sections in the top panels in Fig. 9 and makes a "rough" comparison with the corresponding ones in Fig. 7. The variation with beam energy and nucleon scattering angle is very similar in the two cases, and from this alone it is not clear why the situations with and without FSI differ. To understand clearly why and when FSI cause scaling violations, let us consider the scaling procedure in two steps: (i) dividing the cross sections by the corresponding single-nucleon cross section [see Eq. (2)] and (ii) representing the so-obtained results against the scaling variable. Figure 10 illustrates these two steps for the cases of $20^{\circ}$ and $60^{\circ}$ in the RPWIA. Let us focus first on the top panels, where we show the differential cross sections for ${ }^{12} \mathrm{C}(v, p)$ as a function of the outgoing nucleon kinetic energy for two values of neutrino beam energy. The differences between $20^{\circ}$ and $60^{\circ}$ cross sections are clearly visible. As already mentioned and explained using the free-nucleon case in the previous section, the cross sections at $20^{\circ}$ are subject to a much stronger shift to higher $T_{N}$ values with increasing beam energy than at $60^{\circ}$. Also the magnitude of the cross sections at different beam energies changes more drastically for $20^{\circ}$ than for $60^{\circ}$. If we divide these differential cross sections by the corresponding single-nucleon ones to get the scaling function, but still plot the results against $T_{N}$, we obtain what is shown in the middle panels of Fig. 10. We observe that this simple step already removes the differences in magnitude for the different beam energies, not only for $60^{\circ}$ but also for $20^{\circ}$. If we now represent these results as a function of the scaling variable, the shift observed when changing the beam energy disappears, and scaling of the first kind shows up. Both steps (i) and (ii) must be carried out to bring together the cross sections for different beam energies. We see how in the RPWIA the dependence of the QE neutral-current neutrino cross section on the energy of the outgoing nucleon is well described by its single-nucleon content, and it is consequently canceled out during the scaling analysis.

In what follows we analyze the situation when FSI are included. Figure 11 shows how FSI modify the ${ }^{12} \mathrm{C}(v, p)$ cross sections at $20^{\circ}$ and $60^{\circ}$ for different beam energies. These two angles are representative of the regimes where first-kind scaling is broken (forward angles) or fulfilled (large angles), respectively. One observes that FSI involve a redistribution of strength that depends on the energy of the final nucleon. Values of $T_{N}$ far from each other are affected very differently by FSI. In the case of $20^{\circ}$ this effect is clearly stronger, as the cross sections for the various beam energies span a broader region of nucleon kinetic energies. This extra $T_{N}$ dependence from FSI is not contained in the single-nucleon cross section, and thus the scaling procedure is not enough to compensate for it if the cross sections peak at very different $T_{N}$ values. This is clearly appreciated if we follow steps (i) and (ii) as we have done previously in the RPWIA. The results are shown in Fig. 12. As in the RPWIA, a large amount of the variation in magnitude of the cross sections at different beam energies is also removed when the single-nucleon content is factored out, although clear differences still persist in this case for $20^{\circ}$. Moreover, at $20^{\circ}$ one observes the additional $T_{N}$ dependence of the cross section with respect to the plane-wave results (extended tails), and it consequently shows up even when plotting the results against $\psi^{u}$. For $60^{\circ}$ the behavior of results differs because FSI effects are similar for the different beam energies, given the fact that the cross sections peak at similar $T_{N}$ values. In this case, we can say that FSI effects do not spoil scaling. In general, if the kinematics of the process are such that the range of energies of 

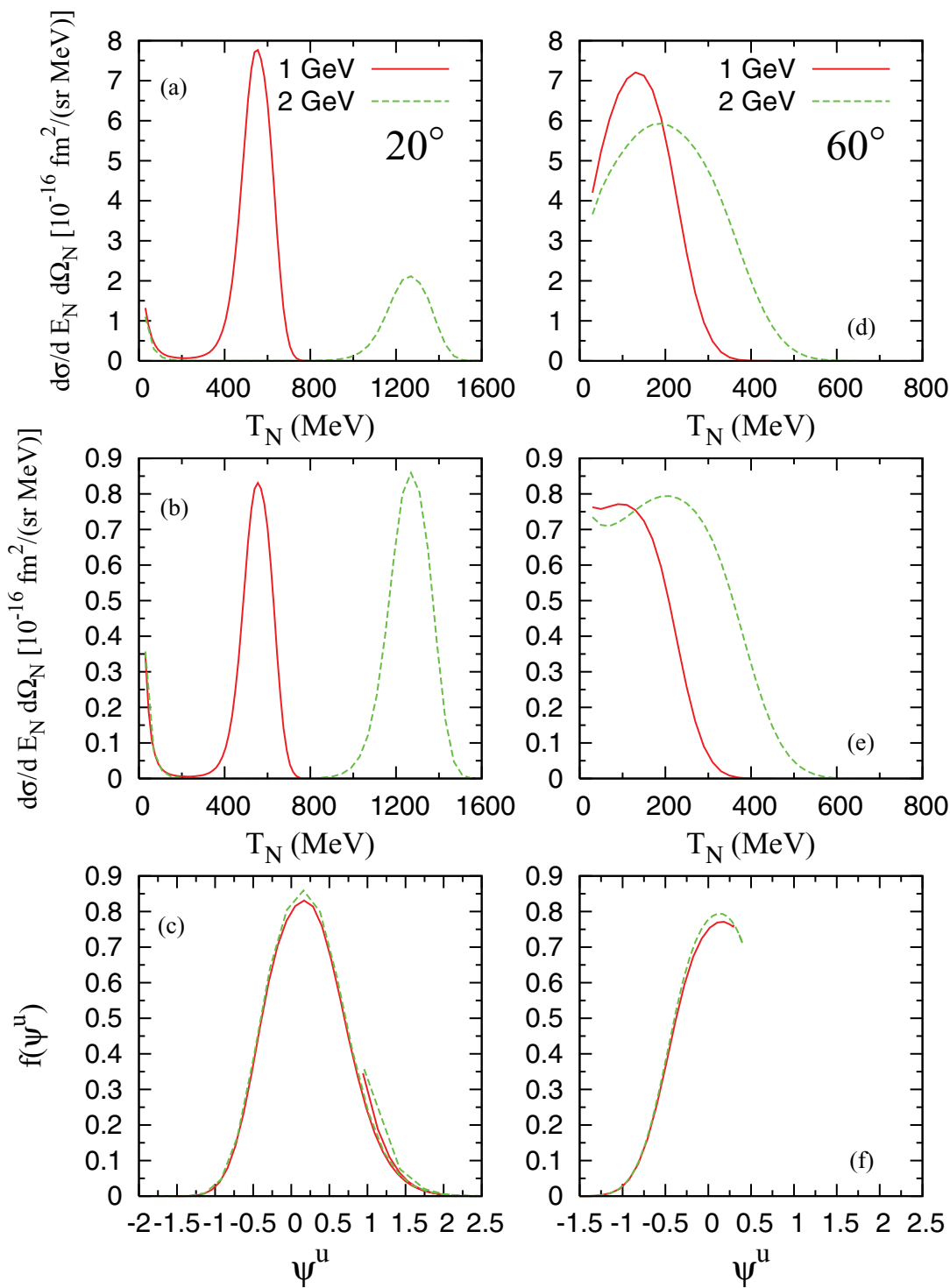

FIG. 10. (Color online) Illustration of the scaling procedure for $\theta_{k p_{N}}=20^{\circ}$ [(a), (b), and (c)] and $\theta_{k p_{N}}=60^{\circ}[(\mathrm{d})$, (e), and (f)] in the RPWIA. Panels (a) and (d) show the differential cross sections against $T_{N}$ for ${ }^{12} \mathrm{C}(\nu, p)$ at two beam energies for each angle. Panels (b) and (e) show the results after factoring out the singlenucleon cross section and making the results dimensionless (namely, the scaling function) but still as a function of $T_{N}$. Panels (c) and (f) show the scaling function plotted against the scaling variable.

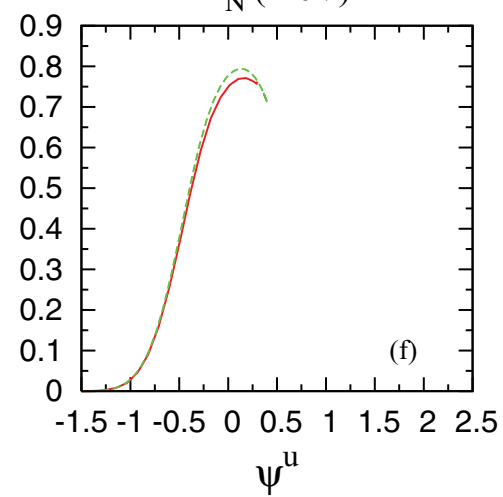

the ejected nucleon depends strongly on the beam energy, the nucleon may be subject to very different FSI for each $\varepsilon$, and a visible breakdown of first-kind scaling will show up. This is what happens in general for forward angles, where there is a strong shift of the position of the peak of the cross section with incoming beam energy. However, for those kinematics for which the range of $T_{N}$ remains approximately the same when considering different beam energies [i.e, for larger $\theta_{k p_{N}}$ (see Fig. 6)], first-kind scaling emerges even with FSI included, as FSI effects on the knockout nucleon are similar for different beam energies. This outcome, namely, almost perfect first-kind scaling for large angles and violations in the case of forward ones, is based on pure kinematical reasoning, and to this extent (and within the impulse approximation) it is independent of the specifics of the model employed. It appears for any model in which FSI are strongly $T_{N}$ dependent, as is the case for the RMF description. One has to realize that the use of the RMF potential provides a stringent scenario for first-kind scaling in the case of QE neutral-current neutrino reactions. If future QE neutral-current neutrino data suggest weaker FSI, which are less dependent on $T_{N}$ than the ones predicted by the RMF, our results imply that in such a case first-kind scaling would work better for smaller angles than shown here, even without any restriction on $\theta_{k p_{N}}$.

Concerning the bivalued nature of the scaling function $f\left(\psi^{u}\right)$, Fig. 9 shows that in the absence of FSI superscaling is a good approximation and the two values of the scaling function for the same $\psi^{u}$ value are nearly equal. When FSI are present (Fig. 7), and if the kinematics prevent superscaling, the bivalued nature of the superscaled function is significantly revealed.

It is very relevant that for the differential cross section integrated over angles, namely $d \sigma / d T_{N}$, the larger contributions come from angles for which scaling works nicely even in the presence of very strong FSI. This is clearly shown from the comparison between $d \sigma / d T_{N}$ in Fig. 13 and the contributing $d \sigma / d T_{N} d \Omega_{N}$ cross sections in Fig. 5. The situation is more favorable for higher beam energies, as in this case the contribution coming from forward angles moves to higher values of $T_{N}$, where the cross section has 


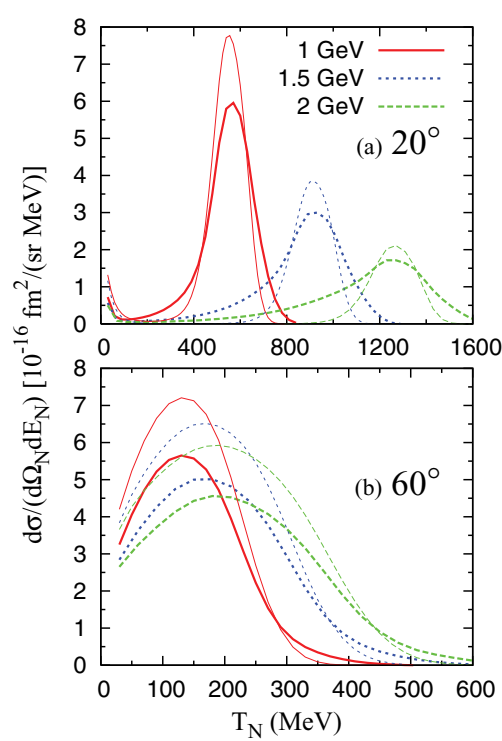

FIG. 11. (Color online) FSI effects in the differential cross sections for ${ }^{12} \mathrm{C}(v, p)$ scattering at three beam energies for (a) $\theta_{k p_{N}}=20^{\circ}$ and (b) $\theta_{k p_{N}}=60^{\circ}$. The thick lines correspond to RMF calculations and the thin ones to the RPWIA. already decreased considerably. Additionally, note that the contribution of forward angles is suppressed in the integration by the phase-space factor $\sin \theta_{k p_{N}}$.

Results of a study of scaling of the second kind are presented in Fig. 14. The scaling function $f\left(q^{\prime}, \psi^{u}\right)$ is evaluated at $\varepsilon=$ $1 \mathrm{GeV}$ for three different targets, ${ }^{12} \mathrm{C}$ as in previous figures, together with ${ }^{16} \mathrm{O}$ and ${ }^{40} \mathrm{Ca}$ with $k_{A}=216$ and $241 \mathrm{MeV} / c$, respectively. These values for the characteristic momentum scale of ${ }^{16} \mathrm{O}$ and ${ }^{40} \mathrm{Ca}$ provide the best superscaling of the data in the case of electron scattering [16]. As in the investigation of first-kind scaling, the values $\theta_{k p_{N}}=20^{\circ}, 40^{\circ}, 60^{\circ}$, and $80^{\circ}$ are considered. The superscaling functions obtained for several nuclei are almost identical (bottom panels), in spite of the strong difference in magnitude of the corresponding cross sections (top panels). That is, the dependence on the nuclear species is well accounted for by the superscaling analysis. Scaling of second kind is seen to be very robust, thereby opening up a means of taking into account nuclear effects for different nuclei by employing superscaling ideas.

In summary, superscaling for QE neutral-current neutrino reactions is fulfilled at the level of $\sim 10 \%$ when very strong FSI are accounted for, provided the angle at which the nucleon is scattered is larger than $50^{\circ}-60^{\circ}$. This $\sim 10 \%$ criterion
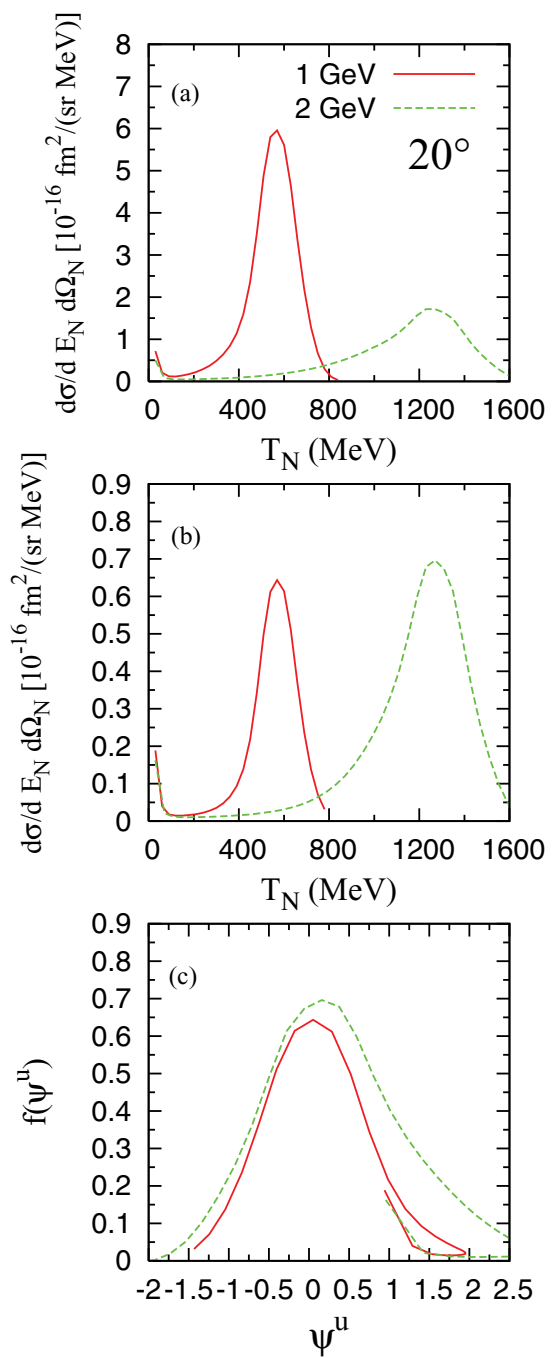
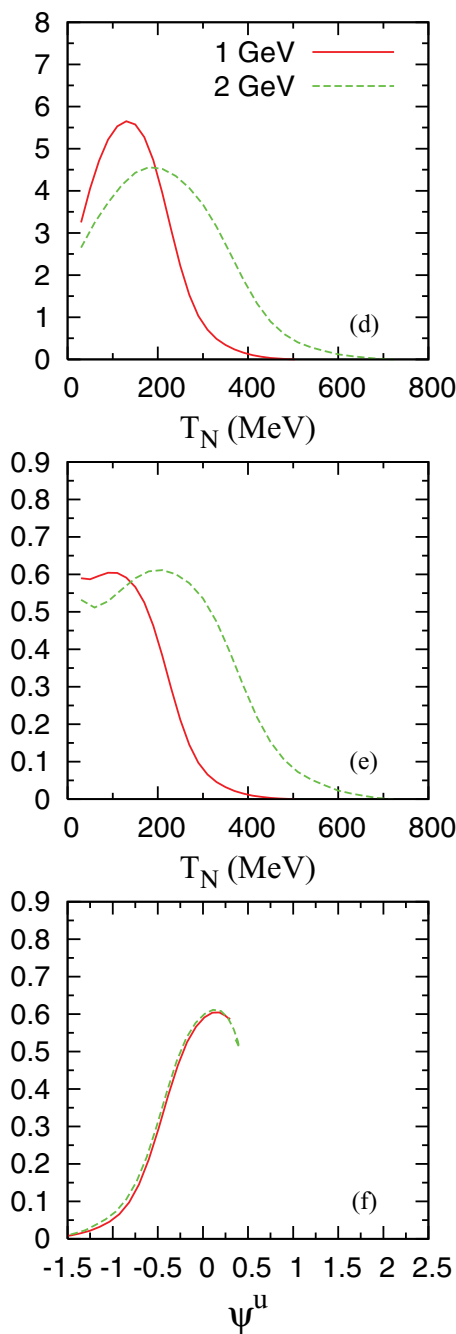

FIG. 12. (Color online) The same plots as in Fig. 10, but now for the RMF. 


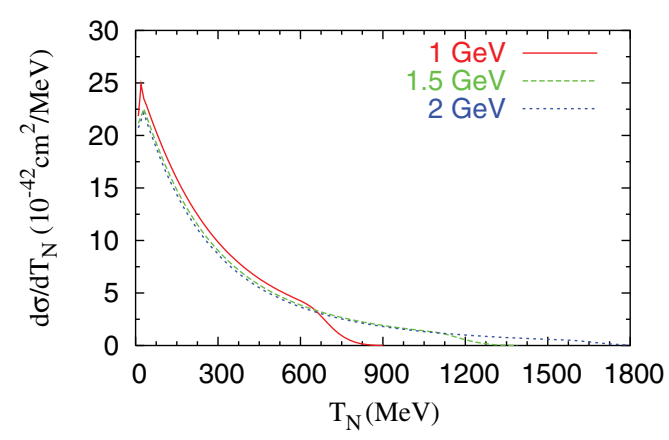

FIG. 13. (Color online) Differential cross section integrated over angles for ${ }^{12} \mathrm{C}(v, p)$ scattering in the RIA-RMF for different beam energies.

corresponds to the maximum difference between the peaks of the scaling functions when dealing with the different neutrino energies, target nuclei, and angles considered in this work.

This analysis has been focused on $(v, p)$ reactions. Although not shown here in detail for the sake of brevity, a similar study has been performed for $(v, n),(\bar{v}, p)$, and $(\bar{v}, n)$ cross sections, and similar conclusions were reached. In spite of the large differences of magnitude and/or behavior observed for cross sections for neutrinos or antineutrinos, and for proton or neutron knockout, the four processes converge to roughly the same superscaling function, with some limitations that are commented upon next. In particular, superscaling functions obtained for protons and neutrons are almost identical in all cases considered in the present work. This is illustrated in Figs. $15(\mathrm{c})$ and $15(\mathrm{~d})$ for scattering from ${ }^{12} \mathrm{C}$ at $1 \mathrm{GeV}$ for
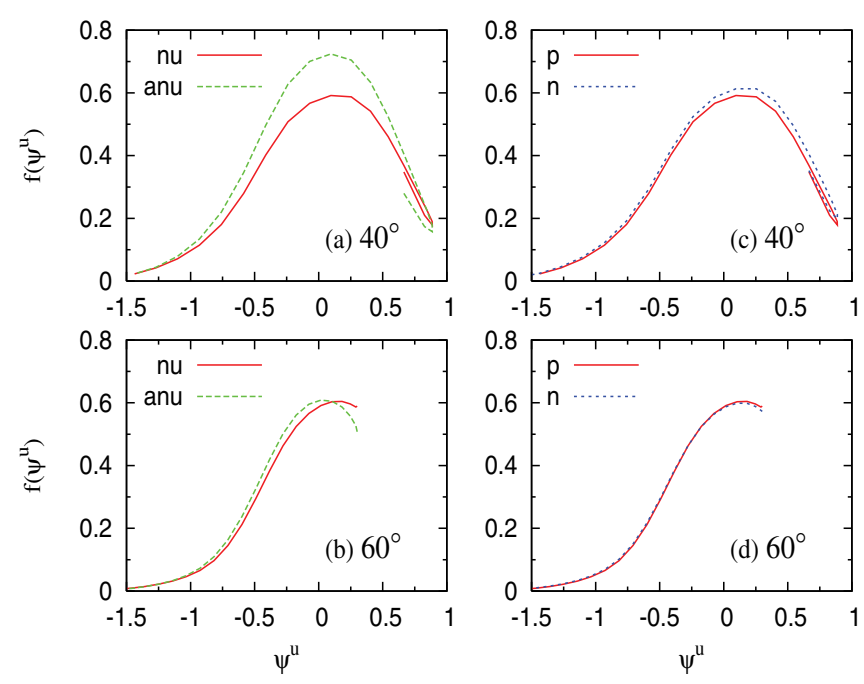

FIG. 15. (Color online) Scaling functions in the RIA-RMF for ${ }^{12} \mathrm{C}$ at $1 \mathrm{GeV}$ for $(v, p)$ vs $(\bar{v}, p)$ in panels (a) and (b) and for $(v, p)$ versus $(v, n)$ in panels (c) and (d). Panels (a) and (c) show results at $\theta_{k p_{N}}=40^{\circ}$, and (b) and (d) correspond to $\theta_{k p_{N}}=60^{\circ}$.

two values of $\theta_{k p_{N}}, 60^{\circ}$ and $40^{\circ}$, that are representative of the kinematical regimes for which first-kind scaling works well (large angles) or is moderately violated (more forward ones). The agreement between superscaling functions obtained for neutrinos and antineutrinos is somewhat worse, as clearly shown in Figs. 15(a) and 15(b). In the case of $60^{\circ}$ there are differences mainly in the region close to the peak of the scaling functions, but overall the two curves are rather
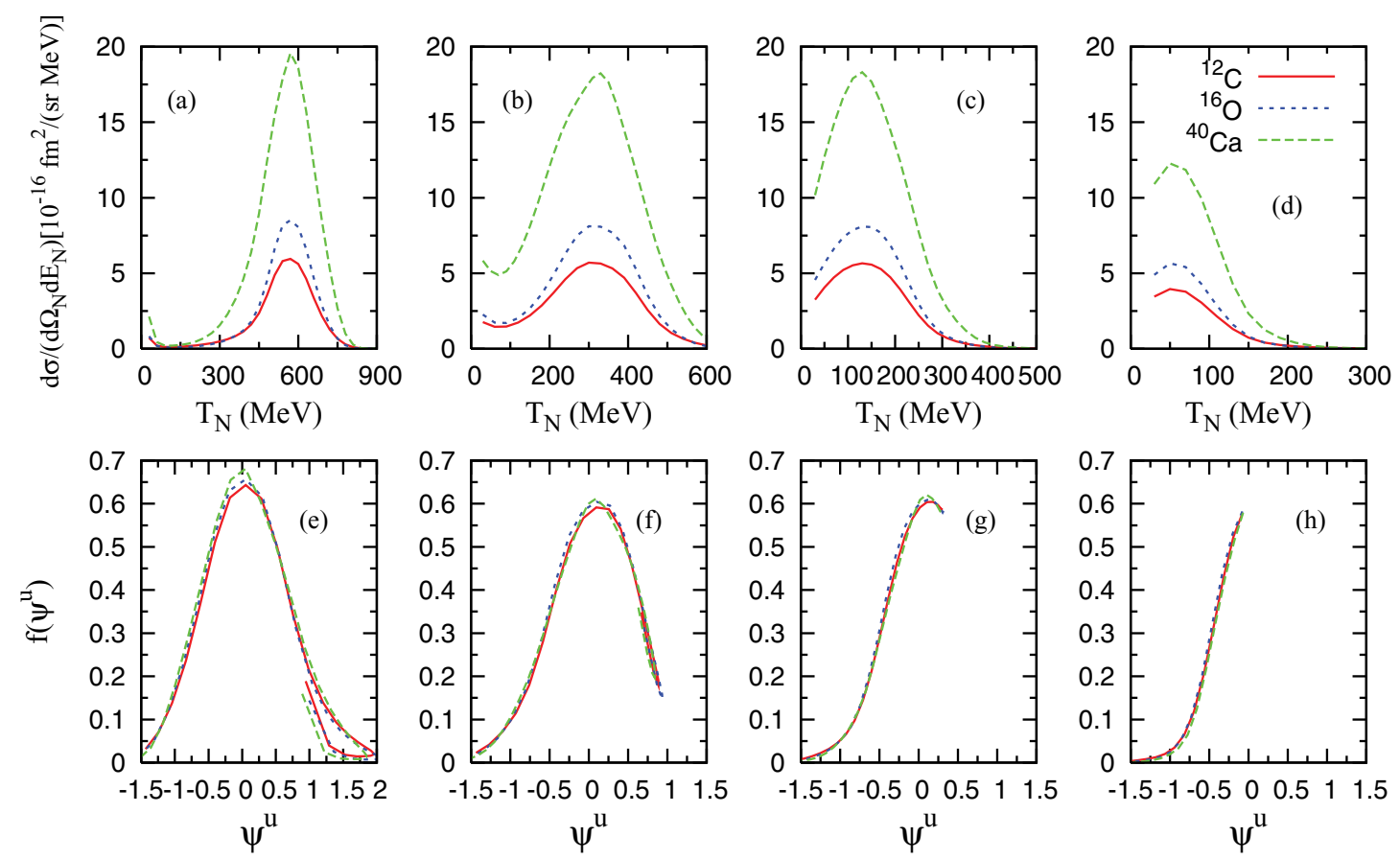

FIG. 14. (Color online) Differential cross section for $(v, p)$ scattering in the RIA-RMF at $1 \mathrm{GeV}$ for different target nuclei [(a), (b), (c), and (d)] and their corresponding scaling functions [(e), (f), (g), and (h)]. As for Figs. 7 and 9, from left to right the graphs correspond to different values of $\theta_{k p_{N}}$, namely $20^{\circ}, 40^{\circ}, 60^{\circ}$, and $80^{\circ}$. 



FIG. 16. (Color online) NC scaling function evaluated within the RIA-RMF approach for (a) $40^{\circ}$ and (b) $60^{\circ}$ at two beam energies, compared with the averaged experimental function, together with a phenomenological parametrization of the $\left(e, e^{\prime}\right)$ data. similar. However, neutrino and antineutrino scaling functions show important deviations for forward angles, for which the first-kind scaling is not well fulfilled. Once more, the reason for this is clearly linked to FSI. We have checked that the $\nu-\bar{v}$ comparison improves considerably in the plane-wave limit.

The exhibition of superscaling by quasielastic neutralcurrent neutrino-nucleus scattering even in the presence of very strong final-state interactions opens the door for investigations of the validity of the universal character of the scaling function for inclusive electroweak processes on nuclei, using either electrons or charged- and neutral-current neutrino probes. To the extent that this universality holds and consequently all of these processes can be described by means of a unique scaling function, the phenomenological SuSA approach formerly applied to predict charged-current neutrino-nucleus cross sections could also provide reliable, largely model-independent, predictions for NC processes.

To study whether or not this universality assumption also holds for NC processes, in Fig. 16 we compare the RIA-RMF $\mathrm{NC}$ superscaling function with the averaged experimental function obtained from the analysis of quasielastic $\left(e, e^{\prime}\right)$ data, together with a phenomenological parametrization $[2,14,16]$. The RIA-RMF superscaling function has been plotted for two values of $\theta_{k p_{N}}$ for which scaling of first-kind is (a) not-so-well fulfilled $\left(40^{\circ}\right)$ or (b) well fulfilled $\left(60^{\circ}\right)$. Results are shown for two beam energies for each angle. As observed, the model gives rise to a $\mathrm{NC}$ scaling function that follows closely the behavior of the $\left(e, e^{\prime}\right)$ function whenever superscaling is well respected $\left(60^{\circ}\right)$. In such a case, it is also noticed that the bivalued behavior of the superscaling function is hardly visible. In contrast, the departure from the $\operatorname{SuSA}\left(e, e^{\prime}\right)$ response is visible for the case of $40^{\circ}$, for which breakdown of first-kind scaling clearly occurs, and the bivalued nature of the $\mathrm{NC}$ superscaled function is enhanced. We notice that all curves would coincide if superscaling were exactly fulfilled in both $\mathrm{NC}$ and $\left(e, e^{\prime}\right)$ cases. Since the $\left(e, e^{\prime}\right)$ and NC scaling curves are obtained under rather different kinematical situations, the scaling curves depart from one another when superscaling is not a good approximation. This supports the assumption that, under proper kinematical restrictions, a universal quasielastic scaling function exists that is valid, not only for inclusive electron and charged-current neutrino reactions as seen in Refs. [3,5], but also for NC processes, making feasible the idea of using the SuSA also to predict NC neutrino-nucleus cross sections.

\section{CONCLUSIONS}

In this paper we have studied the possibility of applying superscaling ideas to NC neutrino scattering processes, which is of interest for experiments that rely on the measurement of neutrino-nucleus cross sections. This is an extension of the work presented in Ref. [22]. The shortage of NC experimental data to date has made mandatory the use of a specific model for this purpose. We have chosen the RIA-RMF approach mainly because of its capability in reproducing the superscaling properties exhibited by inclusive electron data, including the asymmetric tail occurring in the experimental scaling function that has proven to be elusive for most models. Furthermore, the RIA-RMF approach gives rise to scaling violations that are similar to those presented by the electron scattering data. The essential ingredient of the RIA-RMF model that makes this agreement possible is the inclusion of strong FSI between the emitted nucleon and the residual nucleus by means of the same RMF potential already used for describing the initial bound nucleon states. We have only relied on the RIA-RMF model to illustrate possible FSI effects in the superscaling properties of quasielastic neutral-current neutrino cross sections. Our goal was not to explore how this particular model (super)scales or not but rather to determine whether and when the experimental scaling response obtained from electron scattering data can be applied to predict NC neutrino-nucleus cross sections, for which we consider the RIA-RMF model as guidance.

Within the context of the RIA-RMF, we have evaluated inclusive differential cross sections (and separate response functions) for various choices of kinematics and nuclei. The superscaling function has been computed and displayed as a function of the scaling variable $\psi^{u}$ for the different kinematics and various target nuclei. Proceeding in this way, we have investigated scaling of the first and second kinds. From our RIA-RMF results, scaling of the second kind is seen to work extremely well, opening the possibility of accounting for nuclear effects for different nuclei by employing superscaling ideas. With respect to scaling of the first kind, there are kinematics for which scaling is very good and others for which clear scaling violations are observed. We have identified the 
conditions that make a specific choice of kinematics suitable or not for good first-kind scaling: The kinematics must be such that the range of energies spanned by the ejected nucleon depends weakly on the incoming neutrino energy. If the beam energy is given and the angle of the ejected nucleon with respect to the beam is fixed, this situation means angles larger than roughly $50^{\circ}$, which happens to be the region where the cross section integrated over angles reaches larger values. In such cases, first-kind scaling is well respected even in the presence of strong FSI.

We have compared the RIA-RMF superscaling function with the experimental $\left(e, e^{\prime}\right)$ scaling function and have verified that the agreement is good for those kinematics for which first-kind scaling is well fulfilled. This accordance clearly favors the existence of a universal scaling function that is valid for electron and neutrino (charged- or neutral-current) inclusive scattering, and it gives us confidence that, under the kinematical restrictions explained here that ensure good first-kind scaling, the superscaling approach can be extended to predict neutral-current quasielastic neutrino cross sections. We also note that, even though we have illustrated this study within the RIA-RMF model, the kinematical conditions that grant the validity of SuSA are model independent provided the impulse approximation can be safely applied, that is, under quasielastic kinematics with neutrino beam energies from $\sim 500 \mathrm{MeV}$ up to a few GeV. Obviously, there also exist some uncertainties in the assumption of the validity of the (super)scaling hypothesis for neutral currents, mostly because meson-exchange currents have not been considered. Meson-exchange current effects do not scale (see Refs. [36,37]), but they are expected to contribute less than $15 \%$ (see for instance Refs. [38,39]) to these inclusive neutrino-nucleus cross sections at intermediate energies.

\section{ACKNOWLEDGMENTS}

This work was partially supported by DGI (Spain) and FEDER funds FIS2005-01105, FPA2006-07393, FPA200613807, and FPA2007-62216, by the Junta de Andalucía and the INFN-CICYT, and by the Comunidad de Madrid and UCM (910059 "Grupo de Física Nuclear" and PR1/07-14895). It was also supported in part (T.W.D.) by the U.S. Department of Energy Office of Nuclear Physics under Contract No. DE-FG02-94ER40818. M.C.M acknowledges a "Juan de la Cierva" contract from MEC. Computations were performed at the "High Performance Cluster for Physics" funded by UCM and FEDER funds.
[1] Y. Fukuda et al. (Super-Kamiokande Collaboration), Phys. Rev. Lett. 81, 1562 (1998); M. H. Ahn et al. (K2K), Phys. Rev. Lett. 90, 041801 (2003); E. Ables et al. (MINOS), FermilabProposal-0875 (1995); A. A. Aguilar-Arevalo et al. (MiniBooNe), Phys. Rev. Lett. 98, 231801 (2007); I. Ambats et al. (NOvA.), Fermilab-Proposal-0929 (2004); D. Drakoulakos et al. (MINERvA), hep-ex/0405002; S. Brice et al. (FINeSSE), hep-ex/0402007.

[2] J. E. Amaro, M. B. Barbaro, J. A. Caballero, T. W. Donnelly, A. Molinari, and I. Sick, Phys. Rev. C 71, 015501 (2005).

[3] J. A. Caballero, J. E. Amaro, M. B. Barbaro, T. W. Donnelly, C. Maieron, and J. M. Udías, Phys. Rev. Lett. 95, 252502 (2005).

[4] J. E. Amaro, M. B. Barbaro, J. A. Caballero, T. W. Donnelly, and C. Maieron, Phys. Rev. C 71, 065501 (2005).

[5] J. A. Caballero, Phys. Rev. C 74, 015502 (2006).

[6] J. E. Amaro, M. B. Barbaro, J. A. Caballero, T. W. Donnelly, and J. M. Udías, Phys. Rev. C 75, 034613 (2007).

[7] A. N. Antonov et al., Phys. Rev. C 74, 054603 (2006).

[8] M. Martini, G. Co', M. Anguiano, and A. M. Lallena, Phys. Rev. C 75, 034604 (2007).

[9] J. E. Amaro, M. B. Barbaro, J. A. Caballero, and T. W. Donnelly, Phys. Rev. Lett. 98, 242501 (2007).

[10] J. A. Caballero, J. E. Amaro, M. B. Barbaro, T. W. Donnelly, and J. M. Udías, Phys. Lett. B653, 366 (2007).

[11] J. E. Amaro, M. B. Barbaro, J. A. Caballero, and T. W. Donnelly, Phys. Rev. C 73, 035503 (2006).

[12] A. N. Antonov, M. V. Ivanov, M. B. Barbaro, J. A. Caballero, E. Moya de Guerra, and M. K. Gaidarov, Phys. Rev. C 75, 064617 (2007).

[13] D. B. Day, J. S. McCarthy, T. W. Donnelly, and I. Sick, Annu. Rev. Nucl. Part. Sci. 40, 357 (1990).

[14] T. W. Donnelly and I. Sick, Phys. Rev. Lett. 82, 3212 (1999).
[15] T. W. Donnelly and I. Sick, Phys. Rev. C 60, 065502 (1999).

[16] C. Maieron, T. W. Donnelly, and I. Sick, Phys. Rev. C 65, 025502 (2002).

[17] M. B. Barbaro, R. Cenni, A. De Pace, T. W. Donnelly, and A. Molinari, Nucl. Phys. A643, 137 (1998).

[18] M. B. Barbaro, J. A. Caballero, T. W. Donnelly, and C. Maieron, Phys. Rev. C 69, 035502 (2004).

[19] R. J. Peterson, Nucl. Phys. A769, 95 (2006); 769, 115 (2006); 791, 84 (2007); 803, 46 (2008).

[20] W. M. Alberico, A. Molinari, T. W. Donnelly, E. L. Kronenberg, and J. W. Van Orden, Phys. Rev. C 38, 1801 (1988).

[21] M. B. Barbaro, A. De Pace, T. W. Donnelly, A. Molinari, and M. J. Musolf, Phys. Rev. C 54, 1954 (1996).

[22] M. C. Martínez, J. A. Caballero, T. W. Donnelly, and J. M. Udías, Phys. Rev. Lett. 100, 052502 (2008).

[23] J. M. Udías, P. Sarriguren, E. Moya de Guerra, E. Garrido, and J. A. Caballero, Phys. Rev. C 48, 2731 (1993); 51, 3246 (1995); J. M. Udías, J. A. Caballero, E. Moya de Guerra, J. R. Vignote, and A. Escuderos, ibid. 64, 024614 (2001).

[24] W. M. Alberico et al., Nucl. Phys. A623, 471 (1997); Phys. Lett. B438, 9 (1998); Nucl. Phys. A651, 277 (1999).

[25] M. C. Martínez, P. Lava, N. Jachowicz, J. Ryckebusch, K. Vantournhout, and J. M. Udías, Phys. Rev. C 73, 024607 (2006).

[26] C. J. Horowitz and B. D. Serot, Nucl. Phys. A368, 503 (1981); Phys. Lett. B86, 146 (1979); B. D. Serot and J. D. Walecka, Advances in Nuclear Physics, edited by J. W. Negele and E. Vogt, 16 (Plenum Press, New York, 1986), p. 1.

[27] J. A. Caballero, T. W. Donnelly, E. Moya de Guerra, and J. M. Udías, Nucl. Phys. A632, 323 (1998).

[28] J. A. Caballero, T. W. Donnelly, E. Moya de Guerra, and J. M. Udías, Nucl. Phys. A643, 189 (1998). 
[29] C. Maieron, M. C. Martínez, J. A. Caballero, and J. M. Udías, Phys. Rev. C 68, 048501 (2003).

[30] Y. Jin, D. S. Onley, and L. E. Wright, Phys. Rev. C 45, 1333 (1992).

[31] O. Plohl, C. Fuchs, and E. N. E. van Dalen, Phys. Rev. C 73, 014003 (2006).

[32] Y. Horikawa, F. Lenz, and N. C. Mukhopadhyay, Phys. Rev. C 22, 1680 (1980).

[33] C. R. Chinn, A. Picklesimer, and J. W. Van Orden, Phys. Rev. C 40, 790 (1989).
[34] A. Meucci, F. Capuzzi, C. Giusti, and F. D. Pacati, Phys. Rev. C 67, 054601 (2003).

[35] A. Meucci, C. Giusti, and F. D. Pacati, Nucl. Phys. A739, 277 (2004).

[36] J. E. Amaro, M. B. Barbaro, J. A. Caballero, T. W. Donnelly, and A. Molinari, Phys. Rep. 368, 317 (2002).

[37] J. E. Amaro, M. B. Barbaro, J. A. Caballero, T. W. Donnelly, and A. Molinari, Nucl. Phys. A723, 181 (2003).

[38] Y. Umino, J. M. Udías, and P. J. Mulders, Phys. Rev. Lett. 74, 4993 (1995).

[39] Y. Umino and J. M. Udías, Phys. Rev. C 52, 3399 (1995). 